\title{
DENSITOMETRIA ÓSSEA, CTX, NÍVEL DE ATIVIDADE FÍSICA E FORÇA NA PÓS-MENOPAUSA
}

BONE DENSITOMETRY, CTX, LEVEL OF PHYSICAL ACTIVITY, AND STRENGTH IN POSTMENOPAUSAL WOMEN

DENSITOMETRÍA ÓSEA, CTX, NIVEL DE ACTIVIDAD FÍSICA Y FUERZA EN LA POSTMENOPAUSIA

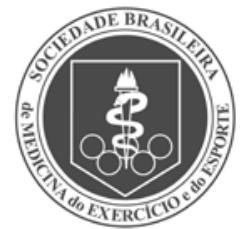

Artigo Original Original ARTICLE Artículo Original
Cristiane Fialho Ferreira da Silva' (Fisioterapeuta)

Paulo Roberto dos Santos Amorim (Educador Físico)

Cristiane Junqueira de Carvalho' (Médica)

Cláudia Loures de Assis ${ }^{\uparrow}$ (Graduanda de Medicina)

Luciana Moreira Lima'

(Bioquímica)

1. Universidade Federal de Viçosa (UFV), Viçosa, MG, Brasil.

\section{Correspondência:}

Luciana Moreira Lima

Universidade Federal de Viçosa, Departamento de Medicina e Enfermagem.

Av. PH Rolfs, s/n. Centro, Viçosa, MG, Brasil. 36570-000.

luciana.lima@ufv.br

\section{RESUMO}

Introdução: O tecido ósseo pode ser avaliado quantitativamente pela densitometria, porém ao associar os biomarcadores ósseos é possível predizer fragilidade óssea mais precocemente. Contudo, pouco se sabe sobre correlação entre ambos, como também sobre o nível de atividade física atual e a força muscular. Objetivo: Avaliar a correlação dos valores da densidade mineral óssea (DMO) aos do biomarcador sanguíneo ósseo (CTX) e também com o nível de atividade física mensurada pelo pedômetro e o questionário IPAQ, versão longa e com os testes de força em mulheres na pós-menopausa. Método: Estudo transversal e descritivo de 62 mulheres na pós-menopausa, saudáveis, média de idade de 56,82 \pm 4,02 anos, avaliadas quanto ao nível de atividade física atual, força, DMO e CTX. Foi realizada densitometria óssea corporal total, de coluna lombar, fêmur e antebraços, além de mensuração de CTX, cálcio iônico, fósforo e PTH. Dois grupos foram formados: controle com DMO normal e grupo com DMO diminuída. Utilizou-se teste t de Student, qui-quadrado e Pearson. Resultados: Não houve diferenças estatisticamente significativas entre os dois grupos para testes de força, nível de atividade física atual e força muscular com relação ao CTX, porém houve associação do valor deste à DMO. Conclusão: Foi demonstrada dependência do CTX com relação aos valores de DMO total e da coluna lombar, ocorrendo baixa DMO quando o CTX estava entre moderado e alto. Isso indica que esse biomarcador talvez possa ser utilizado como forma de triagem de indivíduos com risco de baixa DMO e maior risco de fraturas.

Descritores: densidade mineral óssea; metabolismo; exercício; mulheres.

\section{ABSTRACT}

Introduction: Bone tissue can be assessed quantitatively by densitometry; however, when bone biomarkers are associated, bone fragility can be predicted earlier. However, little is known about the correlation between both, as well as on the current level of physical activity, and muscle strength. Objective: To evaluate the correlation of bone mineral density values (BMD) with the bone blood biomarker (CTX), and also with the level of physical activity measured by pedometer and questionnaire - IPAQ, long version, and the strength tests in postmenopausal women. Method: Descriptive cross-sectional study of 62 postmenopausal, healthy women, with average age of $56.82 \pm$ 4.02, evaluated as for the current level of physical activity, strength, BMD and CTX. Total body densitometry was performed, as well as the lumbar spine, femur and forearms, and the tests for blood CTX, ionic calcium, phosphorus, and PTH. Two groups were formed: control with normal BMD and group with decreased BMD. Student t-test, chisquare, and Pearson were used. Results: There were no significant statistical differences between the two groups for strength tests, current physical activity level and muscle strength in relation to CTX, but there was an association of this value with the values of BMD. Conclusion: CTX has demonstrated dependence in relation to the total BMD and lumbar spine BMD, occurring low BMD when CTX was moderate to high. This indicates that the biomarker might be used as a method of screening individuals at risk for low BMD and increased risk for fractures.

Keywords: bone mineral density; metabolism; exercise; women.

\section{RESUMEN}

Introducción: Se puede evaluar el tejido óseo cuantitativamente por la densitometría, pero al asociar los biomarcadores óseos se puede predecir la fragilidad ósea prematuramente. Sin embargo, poco se sabe acerca de la correlación entre ambos, así como acerca del nivel de actividad física actual y fuerza muscular. Objetivo: Evaluar la correlación de los valores de la densidad mineral ósea (DMO) con los del biomarcador óseo sanguíneo (CTX), como también con el nivel de actividad física mensurable por el podómetro y cuestionario - IPAQ, versión larga y con pruebas de fuerza en mujeres posmenopáusicas. Método: Estudio transversal y descriptivo de 62 mujeres en la postmenopausia, saludables, promedio de edad de 56,82 $\pm 4,02$ años, evaluadas en cuanto al nivel de actividad física actual, fuerza, DMO y CTX. Se realizó densitometría ósea corporal total y de columna lumbar, fémur y antebrazos, además de la medición de CTX, calcio iónico, fósforo y PTH. Se formaron dos grupos: control con DMO normal y grupo con DMO reducida. Se utilizó prueba t de Student, chi-quadrado y Pearson. Resultados: No hubo diferencias estadísticamente significativas entre los dos grupos para las pruebas 
de fuerza, nivel de actividad física actual y fuerza muscular con relación al CTX, pero hubo asociación de su valor con DMO. Conclusión: Se demostró dependencia del CTX con relación a los valores de DMO total y de la columna lumbar, observándose baja DMO mientras el CTX estaba entre moderado y alto. Eso indica que dicho biomarcador quizás pueda utilizarse como un método de clasificación de individuos con riesgo de baja DMO y riesgo aumentado de fracturas.

Descriptores: densidad ósea, metabolismo, ejercicio, mujeres.

\section{INTRODUÇÃO}

O tecido ósseo por ser radiopaco é usualmente avaliado por técnicas de imagens estáticas e pontuais com métodos qualitativos, como as radiografias, e quantitativos, pela densitometria ${ }^{1,2}$. A densitometria óssea por atenuação de raios $X$ de dupla energia (dual energy $X$-ray absorptiometry, DXA) tem sido considerada padrão ouro para avaliação de risco de fraturas em mulheres na pós-menopausa ${ }^{3}$. E, marcadores de reabsorção óssea em níveis elevados têm sido relacionados a altos riscos de fraturas osteoporóticas. Assim, manutenção da densidade mineral óssea (DMO) adequada e baixos níveis desses biomarcadores podem ser considerados fatores de prevenção de fraturas osteoporóticas 4 . A DXA, ainda pouco acessível e de custo elevado, detecta o remodelamento num período mínimo de três a seis meses. Enquanto os biomarcadores retratam o metabolismo ósseo no momento, fornecendo avaliações rápidas, confiáveis e precoces da redução da massa óssea1,2,5. Contudo, estes últimos têm sido mais tradicionalmente utilizados em acompanhamentos de tratamentos ${ }^{6}$ do que propriamente em diagnósticos. Convém ressaltar, os biomarcadores podem se tornar um meio mais eficiente de diagnosticar precocemente problemas do metabolismo ósseo ${ }^{7}$.

O CTX é uma fração carboxi-terminal do colágeno tipo I formada após reabsorção óssea pelos osteoclastos ${ }^{2}$. Seus valores de referência para mulheres em todo o mundo têm sido estudados, principalmente na pós-menopausa, não havendo consenso ${ }^{6}$. Sabe-se que seu valor é influenciado pelo status menopausal, idade, fatores sazonais e ritmo circadiano $2,6,8$.

Pesquisas são desenvolvidas, desde o início da década de sessenta, buscando elucidar os efeitos da atividade física sobre a resistência dos ossos e propondo prescrições de exercícios para sua melhoria e manutenção9. Aoyagi et al. ${ }^{10}$, relacionaram o número de passos diário a redução dos marcadores de reabsorção óssea quando associados à suplementos à base de proteína do leite em mulheres na pós-menopausa. Dore et al. ${ }^{11}$ constataram correlação dos passos diários com a DMO, medida pela DXA, em 740 sujeitos, média de 62 anos, sendo 52\% mulheres na pós-menopausa. Kitagawa et al. ${ }^{4}$ demonstraram associação positiva dos passos diários com a DMO medida pelo ultrassom (US) de calcâneo.

A validade do pedômetro, para mensurar o nível de atividade física, tem sido estudada por muitos autores. Tudor-Locker et al. ${ }^{12}$ observaram que três dias de medição seriam suficientes para se classificar o nível de atividade física habitual de adultos. Outra medida de atividade física, o International Physical Activity Questionnarie (IPAQ), tem sido utilizado em mulheres na pós-menopausa. No estudo de Dallanesi et al. ${ }^{13}$ foi realizado análise da concordância com mulheres na pós-menopausa com osteoporose entre os valores do pedômetro e IPAQ.

Até o momento não foram realizados estudos que identificam a relação do nível de atividade física com a DMO e o CTX na população brasileira. Sendo assim, o objetivo desse estudo foi investigar a correlação dos valores do CTX sanguíneo com a DMO, nível de atividade física habitual e força muscular em mulheres na pós-menopausa.

\section{MÉTODOS}

Foi realizado estudo transversal, descritivo após divulgação no Município de Viçosa, Minas Gerais, Brasil, convidando voluntárias com os critérios de inclusão: idade de 50 a 70 anos, na pós-menopausa (um ano sem menorreia). Os critérios de exclusão foram: história de histerectomia ou ooforectomia, diabetes ou alterações da tireoide, reposição hormonal no último ano, uso de medicamentos que afetem o metabolismo ósseo, fraturas recentes (ao menos um ano), problemas cardíacos, dificuldades na deambulação e cirurgias bariátricas. Após aprovação pelo Comitê de Ética em Pesquisa da UFV, protocolo n- 431.676, foram avaliadas 95 mulheres. Explicados os objetivos e etapas do estudo, todas assinaram o Termo de Consentimento Livre e Esclarecido, formulado segundo normas da resolução 466 / 2012 do Ministério da Saúde para pesquisa com seres humanos, de acordo com a Declaração de Helsinque revisada em 2008. Sete voluntárias desistiram, as demais 88 passaram pelo exame clínico com médico reumatologista, e, 24 apresentaram algum critério de exclusão. Foram incluídas 64 mulheres, porém duas sofreram fraturas durante a pesquisa, totalizando 62 voluntárias. Todas realizaram o registro alimentar de três dias nessa primeira etapa ${ }^{14}$.

Na segunda etapa, as participantes selecionadas responderam ao questionário IPAQ ${ }^{15}$, forma longa, e, realizaram os testes de força:

1) Teste de Preensão de Mão: utilizou-se dinamômetro modelo JAMAR - Sammons Preston, INC Bolingbrook, IL 60440, E.U.A, na postura sentada, ombro aduzido e em rotação neutra, cotovelo fletido a $90^{\circ}$ e antebraço em posição neutra ${ }^{16}$. Foram executadas três repetições de cinco segundos de força máxima, em cada mão, alternadamente, com intervalo de um minuto $^{16}$. Considerou-se o maior valor registrado em kgfl7.

2) Teste de 30 segundos de bíceps: utilizando halter de dois quilos e sentada em cadeira com tronco apoiado no encosto e pés no chão. Mediu-se o máximo de flexões e extensões em 30 segundos com o membro superior direito, e, após um minuto de descanso, com o membro contralateral ${ }^{18}$. Foram registrados somente os movimentos com arco total de flexo-extensão do cotovelo.

3) Teste de sentar e levantar: sentada em cadeira sem braços, tronco apoiado no encosto, pés no chão na largura do quadril e braços cruzados à frente do tórax. Ficava-se de pé e retornava-se à posição inicial o maior número de vezes em 30 segundos ${ }^{18}$.

Em seguida, todas foram orientadas a utilizar o pedômetro (DIGI-WALKER SW 200, Yammax, Japão) por sete dias consecutivos, na cintura, lado direito, na linha do joelho. Sua retirada era permitida para dormirem, banharem ou prática de atividades aquáticas. As classificações dadas foram: "muito ativas" - quando executavam mais que 12500 passos, "ativas" - entre 10000 e 12499, "insuficientemente ativas" - de 5000 a 9999, e,"sedentárias" - abaixo de 500019,20.

$\mathrm{Na}$ terceira etapa, amostras de sangue venoso foram colhidas das sete às nove horas da manhã, após jejum mínimo de oito horas e em abstinência de exercícios intensos por 72 horas ${ }^{1,7,21}$. Mensuraram-se os níveis de concentração sanguíneos de cálcio iônico, fósforo inorgânico, paratormônio e CTX. Para mensuração do CTX, utilizou-se o método de eletroquimioluminescência ${ }^{21}$ no aparelho modular com kit do fabricante Roche ${ }^{\circledR}$. 
As concentrações de PTH foram mensuradas pela eletroquimioluminescência no aparelho modular com kit do fabricante Roche ${ }^{\circledR}$, concentração de fósforo pelo colorimétrico - molibdato de amônio e a concentração de cálcio iônico - ambos medidos com aparelho Cobas Mira Plus da fabricante Bioclin/Quibasa - foi calculado a partir do cálcio total (medido pelo método colorimétrico - arsenazo III), albumina (método colorimétrico - verde de bromocresol) e proteína total (método colorimétrico - biureto).

No mesmo dia, realizou-se densitometria corporal total, da coluna lombar (L1 a L4), fêmur proximal total, colo femoral, antebraço distal total (terço distal) e distal-distal (extremidade distal) bilateralmente. Todos os exames foram realizados pelo mesmo avaliador para garantir consistência das medidas e posicionamentos. Utilizou-se o aparelho Lunar Prodigy Adavance DXA System (analysis version: 13,31) fabricada por GE Healthcare Medical, modelo 8743, Madison, WI, USA. Foi realizada calibração por "phantom" diariamente ${ }^{22,23}$, seguindo recomendações do fabricante. As voluntárias foram classificadas segundo as definições do Consenso Brasileiro de Osteoporose $2002^{24}$, utilizando o T score. E, dois grupos foram constituídos: controle com DMO normal (T score $\geq-1$ ) e grupo com DMO diminuída que compreendeu as classificações de osteopenia e osteoporose (T score $<-1$ ).

\section{Análise estatística}

O tamanho mínimo da amostra foi definido pelo coeficiente de variação previamente descrito para a dosagem de CTX $(46 \%)^{25}$, consi- derando 20\% de variação em torno da média, chegando ao mínimo de 22 indivíduos por grupo ${ }^{26}$ para que fossem demonstradas as possíveis diferenças estatísticas ao nível de significância de cinco por cento.

Os resultados estão descritos em médias e desvios padrões. O teste de Kolmogorov-Smirnov verificou a distribuição normal das variáveis. E contagem dos passos diários em sete dias totais, seis dias (retirando o primeiro), cinco (dias de semana), quatro dias seguidos, três seguidos (dois dias de semana e um de fim de semana - FDS) e dois (somente o FDS), testes de força, DMO e CTX, foram analisados pelo $t$ de Student para amostras independentes. Realizaram-se correlações entre todas as variáveis pelos testes de Pearson e qui-quadrado.

Utilizou-se programa estatístico IBM SPSS versão 20 e nível de significância de $p<0,05$.

\section{RESULTADOS}

Os valores do CTX, na amostra estudada de 62 mulheres na pós-menopausa, variaram de 0,144 a 0,773 ng/mL, com média de 0,398 \pm 0,162 ng/mL. No grupo com DMO normal, levando em consideração a classificação pela densitometria da coluna e fêmur proximal, o valor de CTX foi 0,361 $\pm 0,176 \mathrm{ng} / \mathrm{mL}$ e no grupo com DMO reduzida $0,419 \pm 0,152 \mathrm{ng} / \mathrm{mL}$, porém sem diferenças estatisticamente significativas. Na Tabela 1, estão descritos os valores da DMO por sítio ósseo e os valores do CTX sanguíneo, fósforo, cálcio iônico e PTH nos grupos com DMO normal e diminuída. Houve

Tabela 1. Diferenças nos sítios ósseos entre os grupos com DMO normal e diminuída em relação aos parâmetros sanguíneos.

\begin{tabular}{|c|c|c|c|c|c|c|c|c|c|}
\hline & $\mathbf{N}$ & Cálcio (mg/dL) & $p$ & Fósforo (mg/dL) & $p$ & PTH (pg/mL) & $p$ & CTX (ng/mL) & $p$ \\
\hline DXA lombar & 62 & & 0,296 & & 0,152 & & 0,792 & & 0,169 \\
\hline DMO reduzida & 38 & $4,78 \pm 0,17$ & & $3,85 \pm 0,36$ & & $38,67 \pm 14,76$ & & $0,421 \pm 0,155$ & \\
\hline DMO normal & 24 & $4,73 \pm 0,13$ & & $3,70 \pm 0,44$ & & $37,65 \pm 14,52$ & & $0,363 \pm 0,169$ & \\
\hline DXA fêmur D & 62 & & 0,375 & & 0,891 & & 0,796 & & 0,465 \\
\hline DMO reduzida & 22 & $4,78 \pm 0,17$ & & $3,80 \pm 0,33$ & & $37,62 \pm 14,49$ & & $0,380 \pm 0,118$ & \\
\hline DMO normal & 40 & $4,75 \pm 0,15$ & & $3,79 \pm 0,43$ & & $38,63 \pm 14,76$ & & $0,408 \pm 0,182$ & \\
\hline DXA colo F D & 62 & & 0,157 & & 0,469 & & 0,940 & & 0,650 \\
\hline DMO reduzida & 26 & $4,79 \pm 0,17$ & & $3,84 \pm 0,41$ & & $38,11 \pm 13,24$ & & $0,409 \pm 0,152$ & \\
\hline DMO normal & 36 & $4,74 \pm 0,15$ & & $3,76 \pm 0,39$ & & $38,39 \pm 15,62$ & & $0,390 \pm 0,170$ & \\
\hline DXA fêmur E & 62 & & 0,228 & & 0,893 & & 0,670 & & 0,422 \\
\hline DMO reduzida & 21 & $4,79 \pm 0,16$ & & $3,80 \pm 0,40$ & & $37,16 \pm 12,99$ & & $0,375 \pm 0,124$ & \\
\hline DMO normal & 41 & $4,74 \pm 0,15$ & & $3,79 \pm 0,40$ & & $38,84 \pm 15,41$ & & $0,410 \pm 0,178$ & \\
\hline DXA colo F E & 62 & & 0,824 & & 0,936 & & 0,405 & & 0,349 \\
\hline DMO reduzida & 26 & $4,76 \pm 0,17$ & & $3,80 \pm 0,40$ & & $40,10 \pm 16,23$ & & $0,377 \pm 0,120$ & \\
\hline DMO normal & 36 & $4,76 \pm 0,15$ & & $3,79 \pm 0,40$ & & $36,95 \pm 13,29$ & & $0,414 \pm 0,187$ & \\
\hline DXA rádio $\mathrm{D}$ distal $\mathrm{T}$ & 62 & & 0,393 & & 0,082 & & 0,391 & & 0,886 \\
\hline DMO reduzida & 36 & $4,76 \pm 0,16$ & & $3,87 \pm 0,41$ & & $36,91 \pm 13,49$ & & $0,401 \pm 0,159$ & \\
\hline DMO normal & 26 & $4,74 \pm 0,15$ & & $3,69 \pm 0,36$ & & $40,16 \pm 15,99$ & & $0,394 \pm 0,168$ & \\
\hline DXA rádio D distal d & 62 & & 0,240 & & 0,857 & & 0,769 & & 0,720 \\
\hline DMO reduzida & 37 & $4,74 \pm 0,15$ & & $3,80 \pm 0,43$ & & $37,82 \pm 14,21$ & & $0,404 \pm 0,157$ & \\
\hline DMO normal & 25 & $4,79 \pm 0,16$ & & $3,78 \pm 0,35$ & & $38,94 \pm 15,31$ & & $0,389 \pm 0,172$ & \\
\hline DXA rádio $\mathrm{E}$ distal T & 62 & & 0,857 & & $0,016^{*}$ & & 0,403 & & 0,208 \\
\hline DMO reduzida & 36 & $4,76 \pm 0,16$ & & $3,90 \pm 0,38$ & & $36,95 \pm 13,12$ & & $0,420 \pm 0,165$ & \\
\hline DMO normal & 26 & $4,76 \pm 0,16$ & & $3,65 \pm 0,38$ & & $40,11 \pm 16,42$ & & $0,368 \pm 0,155$ & \\
\hline DXA rádio E distal d & 62 & & 0,279 & & $0,045^{*}$ & & 0,866 & & 0,201 \\
\hline DMO reduzida & 36 & $4,78 \pm 0,18$ & & $3,88 \pm 0,41$ & & $38,01 \pm 13,22$ & & $0,421 \pm 0,155$ & \\
\hline DMO normal & 26 & $4,73 \pm 0,12$ & & $3,68 \pm 0,35$ & & $38,65 \pm 16,48$ & & $0,367 \pm 0,168$ & \\
\hline
\end{tabular}

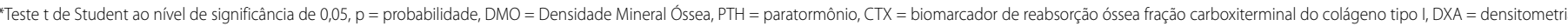
óssea por atenuação de raios $\mathrm{X}$ de dupla energia, $\mathrm{FD}=$ fêmur direito, $\mathrm{FE}=$ fêmur esquerdo, $\mathrm{D}=$ direito, $\mathrm{E}=$ esquerdo, $T=$ total, $d=$ distal. 
diferença estatisticamente significativa somente para os valores do fósforo em relação à DMO do antebraço esquerdo.

No teste de Pearson, não houve correlações do CTX com as medidas de DMO em nenhum sítio ósseo, como também, com os testes de força, e, nem com os resultados do IPAQ.

Na Tabela 2, estão descritos os valores do teste de qui-quadrado para CTX em relação às classificações do T-score. Os valores do qui- quadrado demonstram dependência do CTX em relação ao DMO Total, que leva em consideração a DMO da lombar ou do fêmur proximal total, sendo verificada correlação com $x^{2}=4,406$ ( $p=$ 0,036). Usou-se a classificação do CTX em duas categorias, sendo: 1 - considerado "baixo" com valores até a média 0,398ng/mL e 2 - "moderado a alto" de valores acima da média. E, em relação ao DXA da coluna lombar houve dependência com os valores do CTX observada pelo qui-quadrado de $x^{2}=4,045(p=0,044)$.

Ao dividir o CTX em três categorias (1 - baixo: 0,144 - 0,398; 2 - moderado: 0,399 - 0,573, 3 - alto: acima de 0,574), testou-se a dependência dos valores do mesmo com as classificações dadas pelo pedômetro. Somente as classificações em quatro dias de medições apresentaram associação com o CTX - $(p=0,035)$. (Tabela 3)

Tabela 2. Teste de qui-quadrado para CTX em relação às classificações do T-score.

\begin{tabular}{c|c|c|c|c|c}
\hline & CTX total & $\begin{array}{c}\text { CTX } \\
\text { Baixo }\end{array}$ & $\begin{array}{c}\text { CTX } \\
\text { Médio a Alto }\end{array}$ & X2 & p \\
\hline DXA Total & 62 & $34(54,8 \%)$ & $28(45,2 \%)$ & 4,406 & $0,036^{*}$ \\
\hline DMO reduzida & & $18(29,0 \%)$ & $22(35,5 \%)$ & & \\
\hline DMO normal & & $16(25,8 \%)$ & $6(9,7 \%)$ & & \\
\hline DXA lombar & 62 & $34(54,8 \%)$ & $28(45,2 \%)$ & 4,045 & $0,044^{*}$ \\
\hline DMO reduzida & & $17(27,4 \%)$ & $21(33,9 \%)$ & & \\
\hline DMO normal & & $17(27,4 \%)$ & $7(11,3 \%)$ & & \\
\hline
\end{tabular}

$X^{2}=$ Teste de Qui-quadrado, $P=$ probabilidade * = nível de significancia de 0,05, DMO = Densidade Minera Óssea, CTX = marcador de reabsorção óssea carboxi-terminal do colágeno tipo I, DXA = densitometria óssea por atenuação de raios $X$ de dupla energia.

Tabela 3. Associação entre as classificações dadas pelo CTX e pelo número de passos diários medidos com pedômetro em 4 dias consecutivos.

\begin{tabular}{c|c|c|c|c|c|c|c}
\hline & Sedentário & $\begin{array}{c}\text { Insuficientemente } \\
\text { ativo }\end{array}$ & Ativo & $\begin{array}{c}\text { Muito } \\
\text { ativo }\end{array}$ & Total & X2 & $\mathbf{p}$ \\
\hline CTX baixo & 2 & 20 & 6 & 7 & 35 & & \\
\hline $\begin{array}{c}\text { CTX } \\
\text { moderado }\end{array}$ & 5 & 10 & 3 & 0 & 18 & & \\
\hline CTX alto & 0 & 3 & 4 & 2 & 9 & & \\
\hline Total & 7 & 33 & 13 & 9 & 62 & 13,58 & $0,035^{*}$ \\
\hline
\end{tabular}

2. Teste de Qui-quadrado; *: nível de significância de $0,05, p=$ probabilidade, $C T X=$ marcador de reabsorção óssea carboxi-terminal do colágeno tipo I.

\section{DISCUSSÃO}

O presente estudo não constatou diferenças estatisticamente significativas entre os grupos com DMO normal e DMO diminuída em nenhum sítio ósseo testado em relação ao cálcio iônico, PTH, porém houve diferença em relação ao fósforo. Esse apresentou menor valor no grupo com DMO normal no antebraço esquerdo, sendo essa diferença estatisticamente significativa. Como o fósforo é o segundo elemento constituinte da matriz óssea depois do cálcio, sua menor concentração sérica pode indicar menor atividade reabsortiva dessa matriz. No entanto, esse achado foi presente somente em relação ao antebraço esquerdo e não apresentou outras correlações com os demais exames de CTX, cálcio, PTH, força e nível de atividade física.

Em nosso estudo não houve diferença estatisticamente significante dos valores de CTX no grupo com DMO normal ou diminuída. Contudo, segundo estudos ${ }^{7,21}$ aumento dos marcadores de reabsorção óssea, está associado a risco aproximadamente duas vezes maior de fraturas quando comparadas a mulheres com níveis mais baixos desses marcadores. Brown et al. ${ }^{7}$, observaram que a capacidade do biomarcador em prever fraturas é independente e complementar à medida de DMO. Assim, ao se associar a concentração do CTX com a DMO osteoporótica ou história prévia de fratura tem-se aumento de $70-100 \%$ do risco absoluto de fratura de quadril em comparação à estimativa somente pela DMO baixa ${ }^{7}$. Robbins et al. ${ }^{26}$ tentando explicar porque mulheres com DMO normal também sofriam fraturas frequentes não observaram associação do biomarcador de reabsorção com a DMO. E foi relatado que o aumento deste biomarcador em idosas era mais positivamente associado com risco de fratura na presença de DMO normal do que nas com osteoporose ${ }^{21}$.

No presente estudo houve associação do CTX com a DMO da coluna lombar e com a DMO total, que leva em consideração tanto a DMO do quadril quanto da lombar, amenizando o efeito de alterações na lombar que interfiram na classificação pelo T-score. No estudo de Szulc e Delmas ${ }^{6}$ os biomarcadores foram moderadamente associados à DMO e seu aumento foi associado ao maior risco de fraturas. Porém, essa possível relação é dependente do sítio ósseo mensurado ${ }^{7}$, como citado por Chopin et al. ${ }^{21}$ que relata que a precisão na perda óssea é melhor verificada no quadril do que na coluna lombar, devido a influência da osteoartroses.

No presente estudo observou-se dependência dos valores de CTX com o número de passos diários em quatro dias consecutivos. Foi possível constatar que valores maiores de CTX estavam associados com as voluntárias classificadas como "muito ativas". No entanto, no registro alimentar das participantes verificou-se que a ingestão de cálcio e vitamina D foi deficiente, o que pode ser um fator de estímulo à reabsorção óssea frente à tentativa de se manter a homeostasia do cálcio no organismo. Assim, apesar dos benefícios dos exercícios, no período pós-menopausal, excessos talvez possam trazer potencial risco fragilizando ainda mais o osso ${ }^{27}$. Devine et al. ${ }^{28}$ observaram que mulheres idosas com alto nível de atividade física associados a elevado consumo de cálcio tinham 5,1\% maior DMO do fêmur comparada as com baixos níveis de atividade física e de consumo de cálcio diário. Também, o fato da caminhada ser amplamente recomendada por diferentes órgãos de saúde, pela mídia, sendo considerada atividade natural, segura e acessível, pode ter afetado o comportamento das mulheres com diagnóstico de DMO diminuída, influenciando os resultados obtidos. E, o grupo com maior número de passos apresentou menor peso corporal, fator importante de estímulo através das forças de reação do solo à maior produção de matriz óssea.

Em relação às medidas de força e do nível de atividade física avaliado pelo IPAQ, não houve dependência ou associação com os valores do CTX ou demais variáveis. No estudo de Sun et al. ${ }^{27}$ foi encontrada correlação da força de mão com rigidez óssea medida pelo US em mulheres idosas jovens.

Algumas limitações de nosso estudo foram a não mensuração de marcadores de formação, pois se demonstrou que a manutenção da DMO está mais relacionada ao aumento da formação do que a diminuição da reabsorção óssea ${ }^{29}$. Outra questão é a medida simples 
do marcador, principalmente na fase pós-menopausal, que tem maior variabilidade dos intervalos de referência entre os indivíduos, sendo mais apropriada a medição seriada ${ }^{7}$.

\section{CONCLUSÃO}

No presente estudo foi demonstrada dependência do CTX em relação aos valores de DXA total e da coluna lombar, ocorrendo baixa DMO quando o CTX estava entre moderado e alto. Isso pode indicar que esse biomarcador talvez possa ser utilizado como forma de triagem, antes do DXA, de indivíduos com risco aumentado de fraturas. Como os biomarcadores são usados no acompanhamento de adesão aos tratamentos da osteoporose, os resultados do presente estudo sugerem que também possam ser utilizadas no controle das respostas da atividade física.

\section{AGRADECIMENTO}

Apoio financeiro parcial da Fundação de Amparo à Pesquisa de Minas Gerais (FAPEMIG).

Todos os autores declararam não haver qualquer potencial conflito de interesses referente a este artigo.

CONTRIBUIÇÕES DOS AUTORES: Cada autor contribuiu individual e significativamente para o desenvolvimento do manuscrito. CFFS (0000-0001-6522-3051)* LML (0000-0001-5349-1577)* e PRSA (0000-0002-4327-9190)* elaboraram o design do estudo. CFFS, CJC (0000-0001-8574-6669)* e CLA (0000-0002-6992-7379)* coletaram todos os dados e redigiram a primeira versão do manuscrito. LML e PRSA revisaram o manuscrito e contribuíram na discussão dos resultados e análise estatística. *Numero ORCID (Open Researcher and Contributor ID).

\section{REFERÊNCIAS}

1. Silva CC, Goldberg TB, Nga HS, Kurokawa CS, Capela RC, Teixeira AS, et al. Impact of skeletal maturation on bone metabolism biomarkers and bone mineral density in healthy Brazilian male adolescents. J Pediatr (Rio J). 2011;87(5):450-6.

2. Vieira JGH. Considerações sobre os marcadores bioquímicos do metabolismo ósseo e sua utilidade prática. Arq Bras Endocrinol Metab. 1999;43(6):415-22.

3. García-Pérez MA, Moreno-Mercer J, Tarín JJ, Cano A. Relationship between PTH, sex steroid and bone turnover marker measurements and bone density in recently postmenopausal women. Maturitas. 2003;45(1):67-74

4. Kitagawa J, Nakahara Y. Associations of daily walking steps with calcaneal ultrasound parameters and a bone resorption marker in elderly Japanese women. J Physiol Anthropol. 2008;27(6):295-300.

5. Martínez J, Olmos JM, Hernández JL, Pinedo G, Llorca J, Obregón E, et al. Bone turnover markers in Spanish postmenopausal women: the Camargo cohort study. Clin Chim Acta. 2009;409(1-2):70-4.

6. Szulc P, Delmas PD. Biochemical markers of bone turnover: potential use in the investigation and management of postmenopausal osteoporosis. Osteoporos Int. 2008;19(12):1683-704.

7. Brown JP, Albert C, Nassar BA, Adachi JD, Cole D, Davison KS, et al. Bone turnover markers in the management of postmenopausal osteoporosis. Clin Biochem. 2009;42(10-11):929-42.

8. Filip RS, Zagórski J. Age- and BMD-related differences in biochemical markers of bone metabolism in rural and urban women from Lublin Region, Poland. Ann Agric Environ Med. 2004;11(2):255-9.

9. Schwarz P. Physical activity and bone strength. Scand J Med Sci Sports. 2004;14(1):1.

10. Aoyagi Y, Park H, Park S, Yoshiuchi K, Kikuchi H, Kawakami H, et al. Interactive effects of milk basic protein supplements and habitual physical activity on bone health in older women: A 1-year randomized controlled trial. Int Dairy J. 2010; 20(10):724-30.

11. Dore D, Quinn S, Ding C, Winzenberg T, Jones G. Correlates of subchondral BMD: a cross-sectional study. J Bone Miner Res. 2009;24(12):2007-15.

12. Tudor-Locke C, Burkett L, Reis JP, Ainsworth BE, Macera CA, Wilson DK. How many days of pedometer monitoring predict weekly physical activity in adults? Prev Med. 2005;40(3):293-8.

13. Dallenezi G, Corrente J, Freire B, Mazeto G. Concordância do International Physical Activity Questionnaire com o pedômetro, em mulheres pós-menopausadas portadoras de osteoporose. Rev Bras Clin Med. 2011;9(2):93-6.

14. Fisberg RM, Marchione DML, Colucci ACA. Avaliação do consumo alimentar e da ingestão de nutrientes na prática clínica. Assessment of food consumption and nutrient intake in clinical practice. Arq Bras Endocrinol Metab. 2009;53(5):617-24.

15. Matsudo S, Araújo T, Matsudo V, Andrade D, Andrade E, Oliveira LC, et al. Questionário Internacional de Atividade Física (IPAQ): Estudo de Validade e Reprodutibilidade no Brasil. Rev Bras Ativ Fís Saúde. 2001; 6(2):5-18.
16. Novaes RD, Miranda AS, Silva JO, Tavares BVF, Dourado, VZ. Reference equations for predicting of handgrip strength in Brazilian middle-aged and elderly subjects. Fisioter Pesq. 2009;16(3):217-22.

17. Silva Neto LS, Karnikowiski MG, Tavares AB, Lima RM. Association between sarcopenia, sarcopenic obesity, muscle strength and quality of life variables in elderly women. Rev Bras Fisioter 2012;16(5):360-7

18. Pinto LG, Dias RMR, Salvador EP, Figueira Júnior A, Lima CVG. Effect of the use of elastic bands during water gymnastics classes in muscular strength of women. Rev Bras Med Esporte. 2008;14(5):450-3.

19. Tudor-Locke C, Ainsworth BE, Whitt MC, Thompson RW, Addy CL, Jones DA. The relationship between pedometer-determined ambulatory activity and body composition variables. Int J Obes Relat Metab Disord. 2001;25(11):1571-8

20. Tudor-Locke C, Ham SA, Macera CA, Ainsworth BE, Kirtland KA, Reis JP, et al. Descriptive epidemiology of pedometer-determined physical activity. Med Sci Sports Exerc. 2004;36(9):1567-73.

21. Chopin F, Biver E, Funck-Brentano T, Bouvard B, Coiffier G, Garnero P, et al. Prognostic interest of bone turnover markers in the management of postmenopausal osteoporosis. Joint Bone Spine 2012;79(1):26-31

22. Anijar JR. Densitometria óssea na prática médica. São Paulo: Sarvier; 2003

23. Smith SM, Zwart SR, Heer M, Lee SM, Baecker N, Meuche S, et al. WISE-2005: supine treadmil exercise within lower body negative pressure and flywheel resistive exercise as a countermeasure to bed rest-induced bone loss in women during 60-day simulated microgravity. Bone 2008;42(3):572-81

24. Pinto Neto AM, Soares A, Urbanetz AA, Souza ACA, Ferrari AEM, Amaral B, et al. Concenso Brasileiro de Osteoporose 2002. Rev Bras Reumatol. 2002;42(6):343-54

25. Holecki M, Zahorska-Markiewicz B, Chudek J, Wiecek A. Changes in bone mineral density and bone turnover markers in obese women after short-term weight loss therapy during a 5 -year follow-up. Pol Arch Med Wewn. 2010;120(7-8):248-54

26. Robbins JA, Schott AM, Garnero P, Delmas PD, Hans D, Meunier PJ. Risk factors for hip fracture in women with high BMD: EPIDOS study. Osteoporos Int. 2005;16(2):149-54

27. Sun W, Watanabe M, Tanimoto Y, Kono R, Saito M, Hirota C, et al. Assessment of the best gait parameter in relation to bone status in community-dwelling young-old and old-old women in Japan. Arch Gerontol Geriatr. 2009;49(1):158-61.

28. Devine A, Dhaliwal SS, Dick IM, Bollerslev J, Prince RL. Physical activity and calcium consumption are important determinants of lower limb bone mass in older women. J Bone Miner Res. 2004;19(10):1634-9.

29. Cadore EL, Brentano MA, Kruel LFM. Efeitos da atividade física na densidade mineral óssea e na remodelação do tecido ósseo. Rev Bras Med Esporte. 2005;11(6):373-9. 